\title{
Focusing-schlieren Visualization in A Dual-mode Scramjet
}

\author{
Toshinori Kouchi (Corresponding author) \\ Dept. Mechanical and System Engr., Okayama University \\ Tsushimanaka 3-1-1, Kita, Okyama, Okayama 700-8530, Japan \\ Tell: +81-86-251-8045 / Fax: +81-86-251-8045 \\ E-mail: kouchi@mech.okayama-u.ac.jp
}

Christopher P. Goyne

Dept. Mechanical and Aerospace Engr., University of Virginia 570 Edgemont Rd., Charlottesville, Virginia 22904-4248, USA

Robert D. Rockwell Jr

Dept. Mechanical and Aerospace Engr., University of Virginia 570 Edgemont Rd., Charlottesville, Virginia 22904-4248, USA

James C. McDaniel

Dept. Mechanical and Aerospace Engr., University of Virginia 570 Edgemont Rd., Charlottesville, Virginia 22904-4248, USA 


\begin{abstract}
Schlieren imaging possesses a high sensitivity for measuring density gradients in compressible flowfields, and can be used to capture, not only shock waves and expansion fans but also turbulent structures associated with mixing and wake flows. Conventional schlieren imaging, however, has difficulty clearly capturing such structures in supersonic combustion test facilities. This is because the severe flow temperatures locally change the refractive index of the window glass that is being used to provide optical access. On the other hand, focusing-schlieren imaging presents the potential of reduced sensitivity to thermal distortion of the windows and to clearly capture the flow structures even during a combustion test. This reduced sensitivity is due the technique's ability to achieve a narrow depth of focus. As part of this study, a focusing-schlieren system was developed with a depth of focus near $\pm 5 \mathrm{~mm}$, and was applied to a direct-connect, continuous flow type, supersonic combustion test facility with a stagnation temperature of $1200 \mathrm{~K}$. The present system was used to successfully visualize the flowfield inside a dual-mode scramjet. The imaging system captured combustion induced volumetric expansion of the fuel jet and an anchored bifurcated shock wave at the trailing edge of the ramp fuel injector. This is the first time successful focusing-schlieren measurements have been reported for a dual-mode scramjet.
\end{abstract}




\section{List of Symbols}

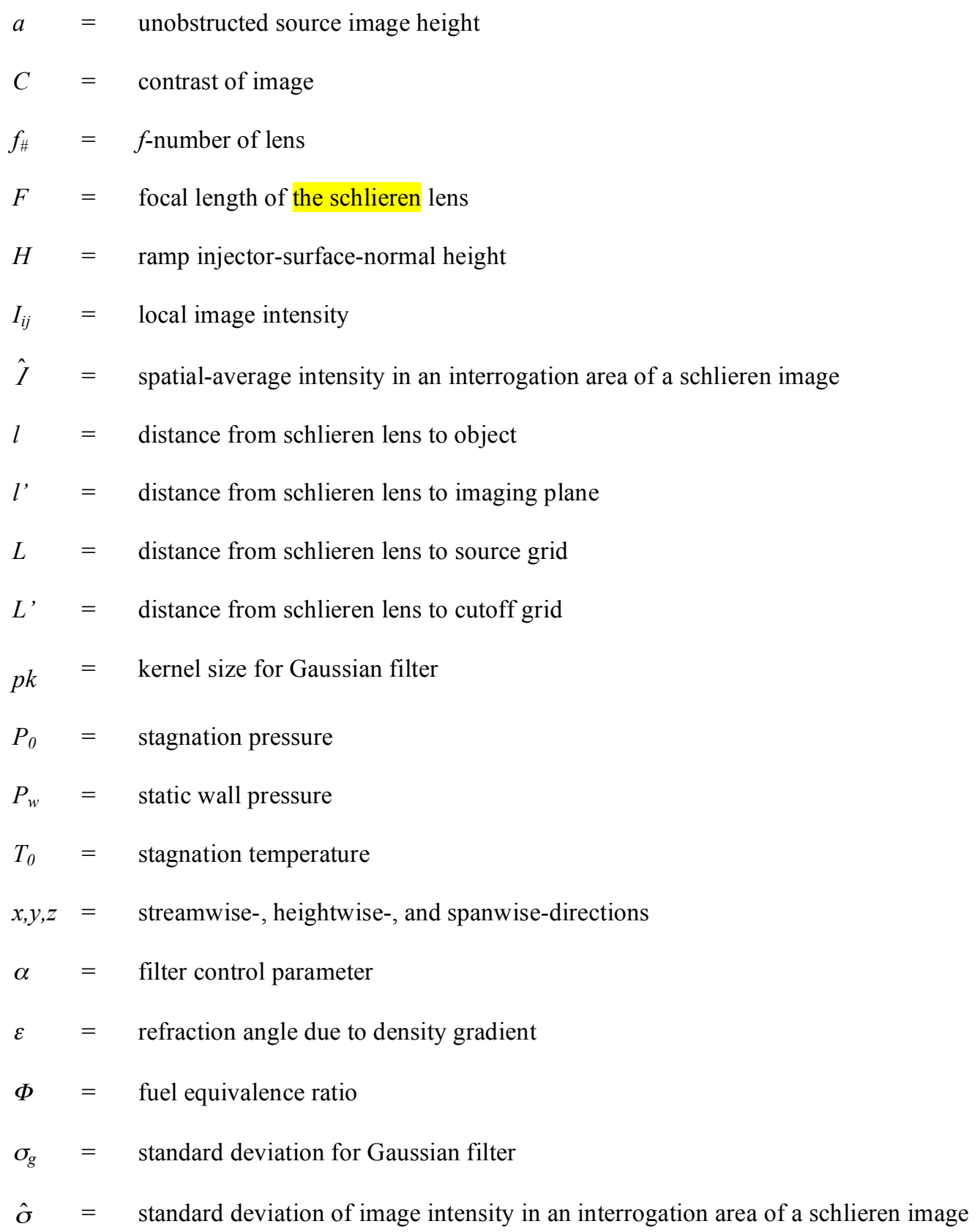




\section{Introduction}

Schlieren imaging is one of the most popular and relatively straightforward flow visualization techniques. This technique has a high sensitivity for measuring density gradients. Therefore, the technique captures not only shock and expansion waves in compressible flows but also turbulent structures within shear layers (Brown and Roshko 1974; Kouchi et al 2009) and boundary layers (VanDercreek et al 2010; Kouchi and Masuya 2012). This technique has been applied to many studies related to scramjet physics: to measure for example, shock-boundary interaction, fuel-air mixing (Ben-Yakar and Hanson 2002; Ben-Yakar et al 2006; Kouchi et al 2009; Kouchi and Mausya 2012), and shock trains (Matsuo et al 1999; Weiss et al 2010).

The technique, however, is rarely applied to supersonic combustion experiments except for those in short duration test facilities (Laurence et al 2011). All supersonic combustion testing requires high enthalpy (temperature) flow to simulate high flight Mach number. The high temperature of the mainstream heats the facility windows that are required for optical access. The refractive index of the window glass is highly dependent on its temperature and is thus locally changed by the heating. The conventional schlieren technique is essentially sensitive to the entire length of the light path including the window glass. As a result, a high enthalpy wind tunnel, with the run times of the order of several seconds or more, generally prevents conventional schlieren from being applied. The window glass exposed to high temperatures distorts the image and induces bright and dark bands in the conventional schlieren images (Fotia and Driscoll 2012). This precludes detailed imaging and prevents complete understanding of the flow structures.

Focusing schlieren is the most promising candidate to avoid such window distortion problems, because the technique has a potential to achieve a narrow depth of focus (DOF) (Settles 2001; Weinstein 2010). Gartenberg et al (1994) applied the focusing-schlieren technique to a cryogenic wind tunnel with low stagnation temperature of about $100 \mathrm{~K}$ and successfully captured the flow structures around a Space Shuttle model. The successful use of the technique, however, has not been reported for dual-mode scramjet flows with high stagnation temperature. The flowfield of a dual-mode scramjet is complex and consists of a three-dimensional mixture of supersonic and subsonic combusting flow (Rockwell et al 2014). In terms of developing a better understanding of the flow, of particular interest is visualizing the uniformity of the scramjet combustor inflow, the compressible turbulent aerodynamics associated with fuel injection and mixing, as well as the 
flame-holding process and the axial progression of combustion as it interacts with the freestream. Because of the relatively long flow establishment time associated with dual-mode scramjets, the flowpaths are typically tested in hypersonic facilities with run times of the order of several seconds or more. This makes the application of schlieren particularly difficult for dual-mode scramjets and, hence, the application is well suited to focusing schlieren.

In the present work, we applied the focusing-schlieren technique to supersonic combustion testing of long duration testing (on the order of hours) and captured the flow structures inside a dual-mode scramjet combustor. The paper begins with a description of the wind tunnel facility and scramjet combustor, including flow conditions. A description of the focusing-schlieren system is then presented before discussing the captured images. Because the minimization of window distortion was a priority for this study, a trade-off analysis, between $D O F$ and system sensitivity, is detailed. This paper represents the first report in the literature of the successful application of focusing-schlieren to a dual-mode scramjet combustion experiment.

\section{Experimental Apparatus}

The experiment was conducted in the University of Virginia Supersonic Combustion Facility (UVaSCF) (Krauss and McDaniel 1992) as part of a broader series of experiments. These experiments are aimed at developing a comprehensive experimental database using advanced optical measurement techniques (Rockwell et al 2012). The UVaSCF is an electrically heated, continuous flow wind tunnel. The facility provided clean air simulating Mach 5 flight enthalpy with a stagnation temperature $\left(T_{0}\right)$ of $1200 \mathrm{~K}$ and a stagnation pressure $\left(P_{0}\right)$ of $300 \mathrm{kPa}$. Figure 1 shows a schematic of a dual-mode scramjet flow path that was directly connected to the Mach 2 facility nozzle. The combustor was $25.4 \mathrm{~mm} \times 38.1 \mathrm{~mm}$ in cross-section at the entrance, and was divided into two sections: a $66.7 \mathrm{~mm}$-long constant-area section and a $402.5 \mathrm{~mm}$-long diverging section. The diverging section had a $2.9^{\circ}$ expansion (on one wall), and terminated with an atmospheric backpressure at the exit of the flowpath. The diverging wall housed an unswept ramp fuel injector. Hydrogen fuel, with a room temperature stagnation condition, was injected through a Mach 1.7 conical nozzle at the base of the ramp with the nozzle axis parallel to the ramp face. The leading edge of the ramp coincided with the onset of the diverging section. The ramp was $12.7 \mathrm{~mm}$ 
wide with the forward face angled $10^{\circ}$ to the diverging wall. The injector-surface-normal height $(H)$ was $6.35 \mathrm{~mm}$; this was used to normalize the spatial variables.

Because the flow static temperature in the combustor was below the auto-ignition temperature of the fuel, measurements could be performed in the case of fuel-air mixing as well as fuel-air reacting. A hydrogen-oxygen detonation driven igniter was used for ignition. The igniter injection port was located on the diverging wall at $x / H \sim 4$. The fuel flow rate was increased to an overall equivalence ratio $(\Phi)$ of 0.2 for successful ignition. Following ignition, the fuel flow rate was decreased to $\Phi=0.17$. Once ignition occurred, combustion was self-sustaining even for $\Phi=0.17$. After achieving a steady-state condition, measurements were performed in the case of fuel-air reacting. For the ignition procedure, measurements were also performed to capture the ignition process. In addition, measurements were performed without fuel injection for various $T_{0}$ conditions during the facility warm-up stage, to investigate the effect of $T_{0}$, particularly due to window distortion.

All components of the combustor, except the windows, were made of stainless steel. The internal walls of the combustor section, including the ramp, were coated in a $0.38 \mathrm{~mm}$ thick layer of thermal barrier zirconia. The wall, housed the ramp injector, had 46 low frequency pressure taps. They were mostly aligned on the combustor centerline. Figure 2 plots the wall pressure $\left(P_{W}\right)$ distributions in the cases of fuel off and fuel-air reacting with $\Phi=0.17$ at $T_{0}=1200 \mathrm{~K}$. Figure 2 also plots $P_{W}$ without fuel injection at $T_{0}=300 \mathrm{~K}$ (open symbols). All $P_{W}$ were normalized by $P_{0}$. Note that $P_{0}$ for the cold flow condition was lower than for the hot conditions because of the restriction on airflow supply rate of the facility.

Referring first to the fuel off distributions, the flow stagnation temperature had no effect on $P_{W}$ upstream of $x / H=15$. The wall pressure on the ramp surface $(x / H=-4.6)$ increased due to the shock wave from the leading edge of the ramp and decreased on the ramp base $(x / H=0)$ due to the expansion waves from the ramp corner. Reflection of these waves induced the fluctuation of $P_{W}$ in the diverging section. The pressure in the diverging section increased at $x / H>15$ for $T_{0}=300 \mathrm{~K}$ due to a terminal shock train. Inviscid quasi-one-dimensional analysis predicts an exit pressure of $P_{e} / P_{0} \sim 0.05$, which corresponds to $P / P_{\text {atm }}=0.11$. This value is below the separation limit to the ambient back pressure (Babinsky \& Harvey 2011). This separation in turn induced the terminal shock train at $x / H>15$. After the facility warm-up, the onset of the shock train was located a further downstream, because $P_{0}$ was higher in this case due to facility considerations. 
Once the fuel-air mixture was ignited, combustion led to a significant pressure rise downstream of the ramp. The wall pressure in the diverging section monotonically increased up to the exit of the combustor and reached the atmospheric backpressure. No pressure rise extended upstream of the ramp. This indicated that the inflow just upstream of the ramp was supersonic in a one-dimensional sense. Even though the combustor flow is a three-dimensional mixture of supersonic and subsonic flow, the combustor was also supersonic in a one-dimensional sense. Therefore, this mode of operation is referred to as the scramjet mode.

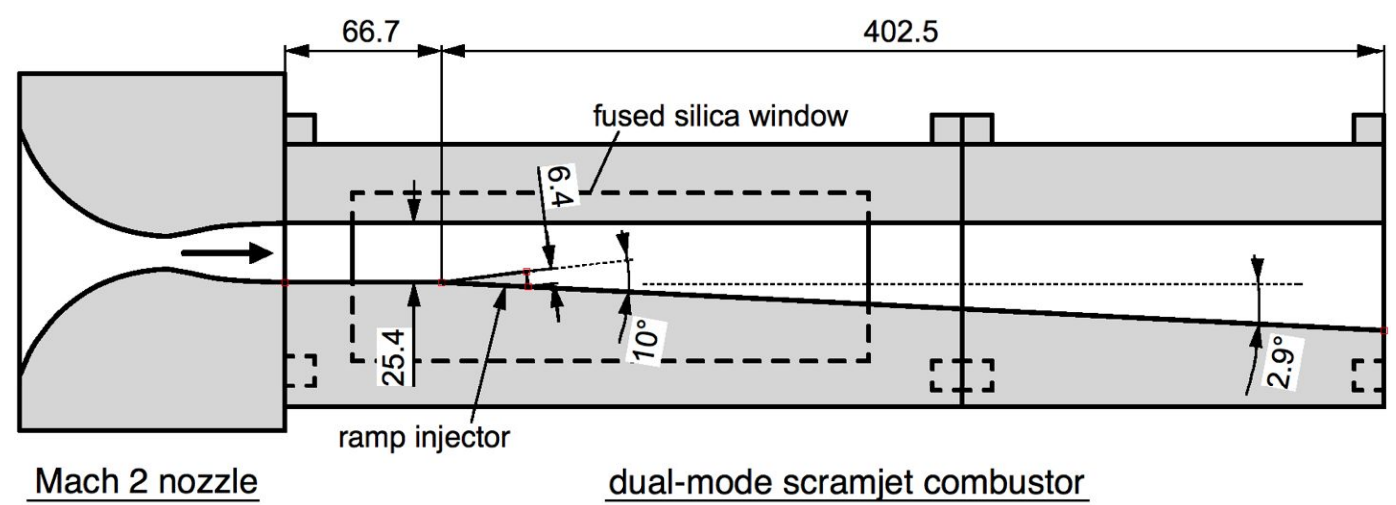

Fig. 1 Schematic view of direct-connect dual-mode scramjet with a ramp injector.

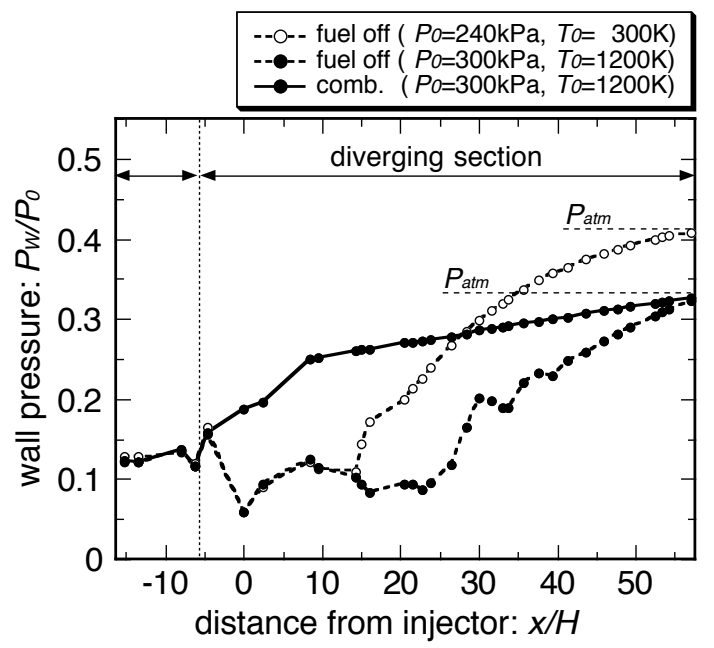

Fig. 2 Wall pressure distribution for cold flow and high temperature combustion cases (typical experimental uncertainty $\pm 2 \%$ ) . 


\section{Focusing-schlieren System}

\subsection{Optical Layout}

As depicted in Figure 1, the scramjet combustor contained two large fused silica windows on the sidewalls for the optical access. The windows were exposed to the high temperature conditions for the test condition at $T_{0}=1200 \mathrm{~K}$. Temperatures on the surface of the glass were expected to reach the adiabatic wall temperature without combustion and to reach the adiabatic flame temperature with combustion. Finite element thermal-structural analyses (Rockwell et al 2012) predicted that about a $200 \mathrm{~K}$ temperature gradient occurred across the thickness of the windows and about $400 \mathrm{~K}$ temperature gradient appeared along the internal surface of the glass. This changed the refractive index of the glass and disabled trials of conventional schlieren.

Figure 3 shows a schematic layout of the focusing-schlieren system for the UVaSCF. Weinstein's modern focusing-schlieren system (Weinstein 2010), using Fresnel lenses and a commercial 35-mm camera lens, was applied. The system consisted of illuminator and analyzer assemblies. The illuminator assembly consisted of a beam diffuser, Fresnel lens and a source grid. The analyzer assembly consisted of a commercial 35-mm camera lens (schlieren lens), a cutoff grid, another Fresnel lens and a CCD camera with an imaging lens.

The light source was a frequency-doubled Nd:YAG laser $(532 \mathrm{~nm})$ with a $10 \mathrm{~ns}$ pulse width and $5 \mathrm{~mJ} /$ pulse energy. This short duration light pulse essentially captured the instantaneous structure of the flowfield. White polypropylene sheets diffused the laser beam and made an extended light source. Coherent illumination usually induces coherent artificial noise on the image. To reduce the coherent noise, three sheets were stacked with a $1 \mathrm{~mm}$ air gap as the beam diffuser. The extended light source was condensed by doubled-Fresnel lens to increase the light-collection efficiency. The diffused beam illuminated a source grid that consisted of multiple, alternating dark band and clear aperture, to make two-dimensional light source array. The dark bands were spaced at the same distance as the clear aperture of $2 \mathrm{~mm}$. The source grid was photographically made and was placed to emphasize density gradients in the streamwise direction.

A commercial $35-\mathrm{mm}$ lens (85 mm-focal length and $1.4 f$-number) was placed at $110 \mathrm{~mm}$ from the object plane. This lens formed both the images of source grid and object on the combustor center plane in each plane to optically conjugate to them. The cutoff grid was placed on the plane where the source grid image was focused. It was adjusted to obstruct a fraction of the 
light from the source grid. The cutoff grid was made by photographically exposing and developing a negative image of the source grid on high-contrast lithographic film. The lithographic film yielded high extinction ratio on the cutoff plane, resulting in high sensitivity of the system.

Another Fresnel lens was placed on the imaging plane in the analyzer assembly. This lens relayed the light from the cutoff grid to the CCD camera. The imaging plane was 374-mm far away from the second principal point of the schlieren lens, because the schlieren lens was placed at $110 \mathrm{~mm}$ from the object plane. This was particularly critical for the present study as this layout achieved a very narrow $D O F$, but magnified the image size of the object on the imaging plane. The image magnification on the Fresnel lens was 3.4 in the analyzer assembly. The image size was approximately $110 \mathrm{~mm} \times 110 \mathrm{~mm}$ for a $30 \mathrm{~mm} \times 30 \mathrm{~mm}$ field of view. The CCD sensor was too small to directly capture the resulting image. Therefore, the image was projected on the Fresnel lens and was relayed to the CCD camera with a large-aperture imaging lens $(55 \mathrm{~mm}$-focal length and $1.2 f$-number). This Fresnel lens was used as not only a screen but also a field (or relay) lens. The Fresnel screen achieved high spatial image resolution in addition to high light transmission, compared with a commonly used ground-glass screen. The CCD camera provided a $1600 \times 1200$ pixel resolution with 14 bit pixel depth at $10 \mathrm{~Hz}$. An optical band pass filter was installed in front of the imaging lens to transmit a 532-nm laser beam and to block both the chemiluminescence of the flame and the glow from the zirconia coating.

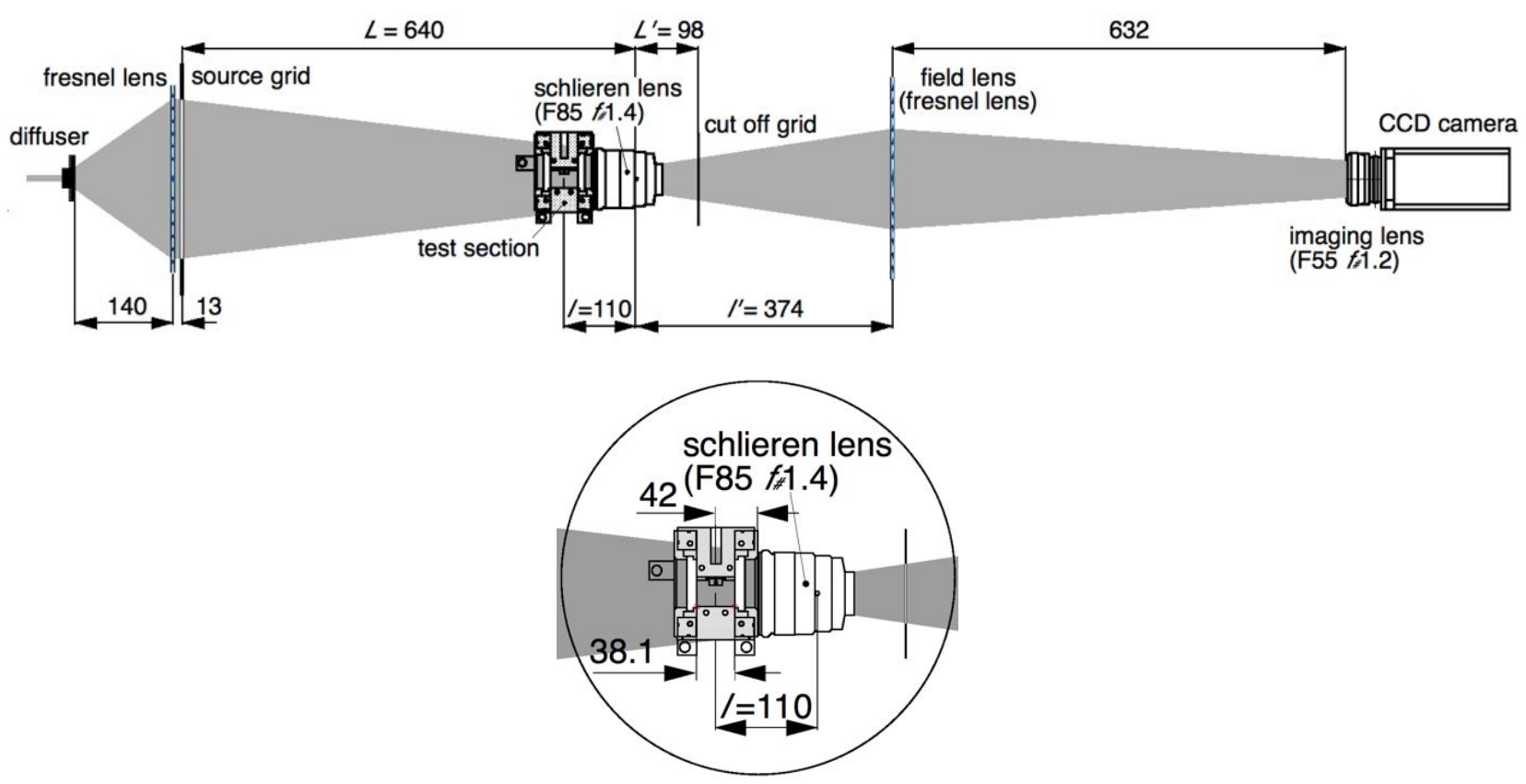

Fig. 3 Schematic layout of focusing-schlieren setup (linear units $\mathbf{m m}$ ). 


\subsection{Sensitivity and Depth of Focus}

As mentioned above, minimization of window distortion was a priority for this study. Therefore, a trade-off study between $D O F$ and sensitivity was performed. The contrast sensitivity of the focusing-schlieren system (Weinstein 2010) is written as follow:

$$
\frac{\partial C}{\partial \varepsilon}=\frac{F}{a}\left(\frac{1-l / L}{1-F / L}\right)
$$

where $C$ is contrast of the image, $\varepsilon$ is refraction angle due to density gradient on the object plane, $F$ is the focal length of the schlieren lens, $a$ is unobstructed height of source grid image on the cutoff plane, $L$ is the distance from the schlieren lens to the source grid, and $l$ is the distance from the schlieren lens to the object plane. On the other hand, the DOF is proportional to $f$-number $\left(f_{\#}\right)$ and $l$, and inversely proportional to $F$. Weinstein (2010) defined the "unsharp" DOF as the region beyond which a predetermined-length-scale structure is effectively blurred due to it being out of focus as follow:

$$
D O F=2 \delta \cdot f_{\#} \frac{l}{F}
$$

where $\delta$ is an acceptable diameter of blurry image, usually $2 \mathrm{~mm}$. A narrow $D O F$ requires a small $f$-number lens which means a large aperture lens. To capture an object as a real image, $l$ cannot be $<F$. An assumption of $l / F=1$ is good approximation for the system having a minimum $D O F$. This assumption converts Eqs. 1 and 2 into

$$
\begin{aligned}
\frac{\partial C}{\partial \varepsilon} & =\frac{F}{a} \\
D O F & =2 \delta \cdot f_{\#}
\end{aligned}
$$

From Eqs. 3 and 4, high sensitivity and narrow $D O F$ imaging system requires a large $F$, small $f$ number schlieren lens. Generally, a high quality large $F$ and small $f_{\#}$ lens is difficult to make and obtain, because this type lens increases optical aberration. Therefore, a trade must take place when designing the system in terms of the sensitivity and $D O F$.

Given this trade-off, a narrower $D O F$ took priority over higher sensitivity for the present system in order to minimize window distortion. For the preliminary experiment, we used the focusing schlieren system using a $300 \mathrm{~mm}$-focal length and $2.8 f$-number lens. The preliminary system had unsharp DOF of $\pm 20 \mathrm{~mm}$ for $\delta=2 \mathrm{~mm}$. This was similar to the width of the test 
section. The system, however, was not able to capture the detailed flow structures in the combustor after the facility warm-up. The preliminary $D O F$ was not enough to avoid the window distortion following the warm-up. Therefore, we carefully redesigned the system having minimum $D O F$ for the UVaSCF. The minimum $l$ is restricted by the facility, because the lens is unable to be placed inside the test section. For the UVaSCF, it is impossible to place the lens closer than $42 \mathrm{~mm}$ from the center plane of the test (section as shown in Fig. 3b). Therefore, $l$ and $F$ must be $>42 \mathrm{~mm}$. On the other hand, a minimum $f_{\#}$ is about 1.2 for an available commercial lens. We selected a lens having $85 \mathrm{~mm}$-focal length and $1.4 f$-number as the schlieren lens for the present system. The lens was positioned as close to the tunnel window as possible. The unsharp $D O F$ was calculated to be \pm $3.6 \mathrm{~mm}$ for $\delta=2 \mathrm{~mm}$ for the present system. Other important specifications of the present focusingschlieren system are calculated and listed in Table 1.

The depth of focus was experimentally confirmed using a 1-mm-diameter, under-expanded supersonic air jet under unheated condition. Figure 4 shows the focusing schlieren images obtained at various object positions. The shock diamonds in the jet were clearly visible in the plane-of-best-focus $(0-\mathrm{mm})$. On the other hand, the shock diamonds were blurred in the $\pm 5-\mathrm{mm}$ planes away from the plane-of-best-focus. In the \pm 10 -mm planes, the shock diamonds were no longer distinguishable from the background. Figure 5 shows the image intensities along the jet center for the various object planes. They were corrected for the background non-uniformity by dividing a quiescent image pixel by pixel to the flow image. The signal peaks associated with each shock cell decreased with the image plane away from the plane-of-best-focus, because the shock structures were blurred by the focusing effect of the imaging system.

To quantify $D O F$, each of the signal peaks was divided by those on the plane-of-best-focus and plotted in Fig. 6. Figure 6 shows that the signal intensities were distributed along a Gaussian distribution with their variance of $3 \sim 5$. The signal intensity became about half, when the object was positioned at \pm 5 - $\mathrm{mm}$ from the plane-of-best-focus which was below the ramp width. When the object was positioned over \pm 10 -mm from the plane-of-best-focus, the signal was similar level to the background noise. The windows were positioned at $\pm 19.05 \mathrm{~mm}$ from the center plane of the combustor. Therefore, the window was completely out of focus for the dual-mode scramjet experiment. This is regarded as a key-enabling factor in the present application. 


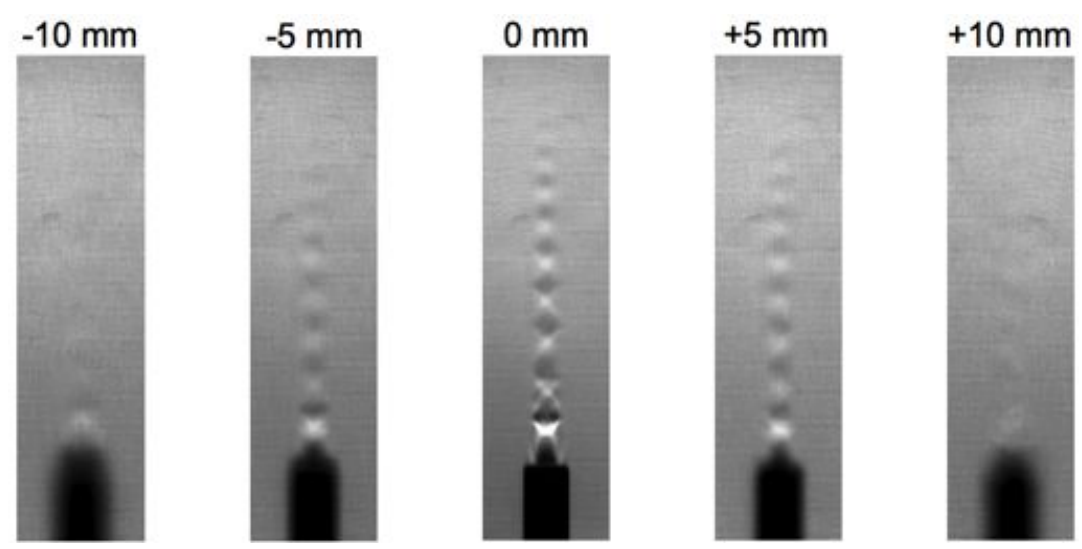

Fig. 4 Experimental demonstration of $D O F$ for the present system. Tube inner diameter was $1 \mathrm{~mm}$, and its outer diameter was $1.6 \mathrm{~mm}$. Time average images for air injection.

Table 1 Specification of focusing-schlieren system.

\begin{tabular}{clc}
\hline \hline $\begin{array}{c}\text { Unobstructed source image height * } \\
\text { Detectable light deflection angle } \\
\text { with 10\% contrast }\end{array}$ & $: \varepsilon_{\text {min }}$ arcsec & 17 \\
Sharp depth of focus & $: D S \mathrm{~mm}$ & \pm 0.5 \\
Unsharp depth of focus ** & $: D U \mathrm{~mm}$ & \pm 3.6 \\
Diffraction limit of resolution & $: \omega \mathrm{mm}$ & 0.28 \\
No. of gridlines in cut-off grid & $: N \mathrm{~mm}{ }^{-1}$ & 1.6 \\
No. of blended images & $: \phi$ & 36 \\
\hline \hline
\end{tabular}

* Assuming $a=2 /(30$ line-pairs/mm lens resolution)

** Assuming circule of confusion $=2 \mathrm{~mm}$.

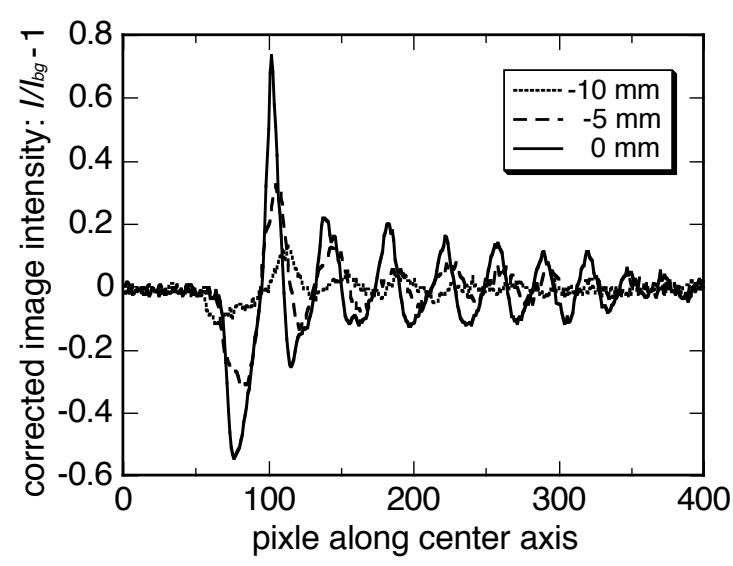

Fig. 5 Corrected image intensity in underexpanded jet for various object planes.

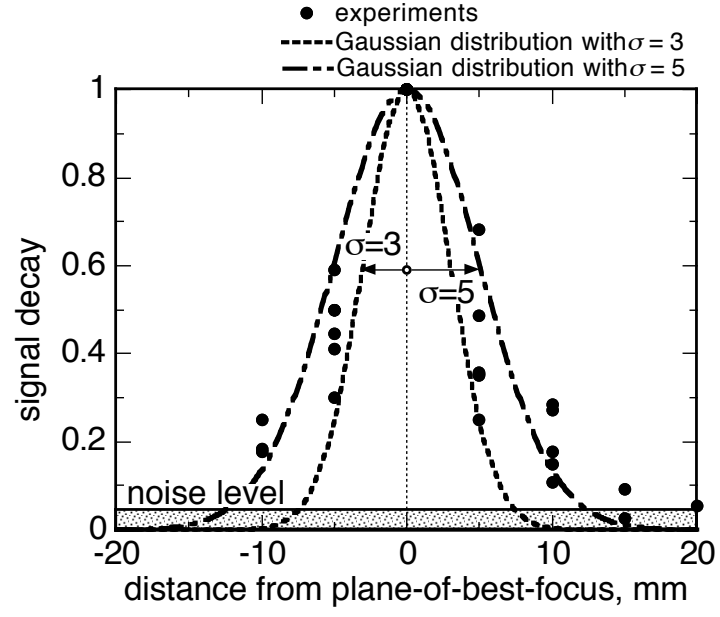

Fig. 6 Decay of signal intensity in shock cells away from the best-of-focus-plane. 


\section{$4 \quad$ Image Processing}

The acquired images were post-processed to enhance the shock waves and turbulent structures in each image. Figure 7 shows the flowchart of the image processing used in this study. This image processing mainly consists of shade correction, noise reduction and image deformation parts. In the shade correction part, nonlinear unsharp masking filter corrected the uneven illumination of the background on the original image and enhanced the flow structures in the images. The filter enabled a better identification of the flow features. This filter enhanced not only flow structures but also the concentric circle fringes on the Fresnel lens as used for the screen of the analyzer system. Therefore, a low pass frequency filter was applied to eliminate the Fresnel pattern in the noise reduction part. In the image deformation part, the images were dewarped to correct the lens distortion of the imaging system. Details on each process are discussed in the following section.
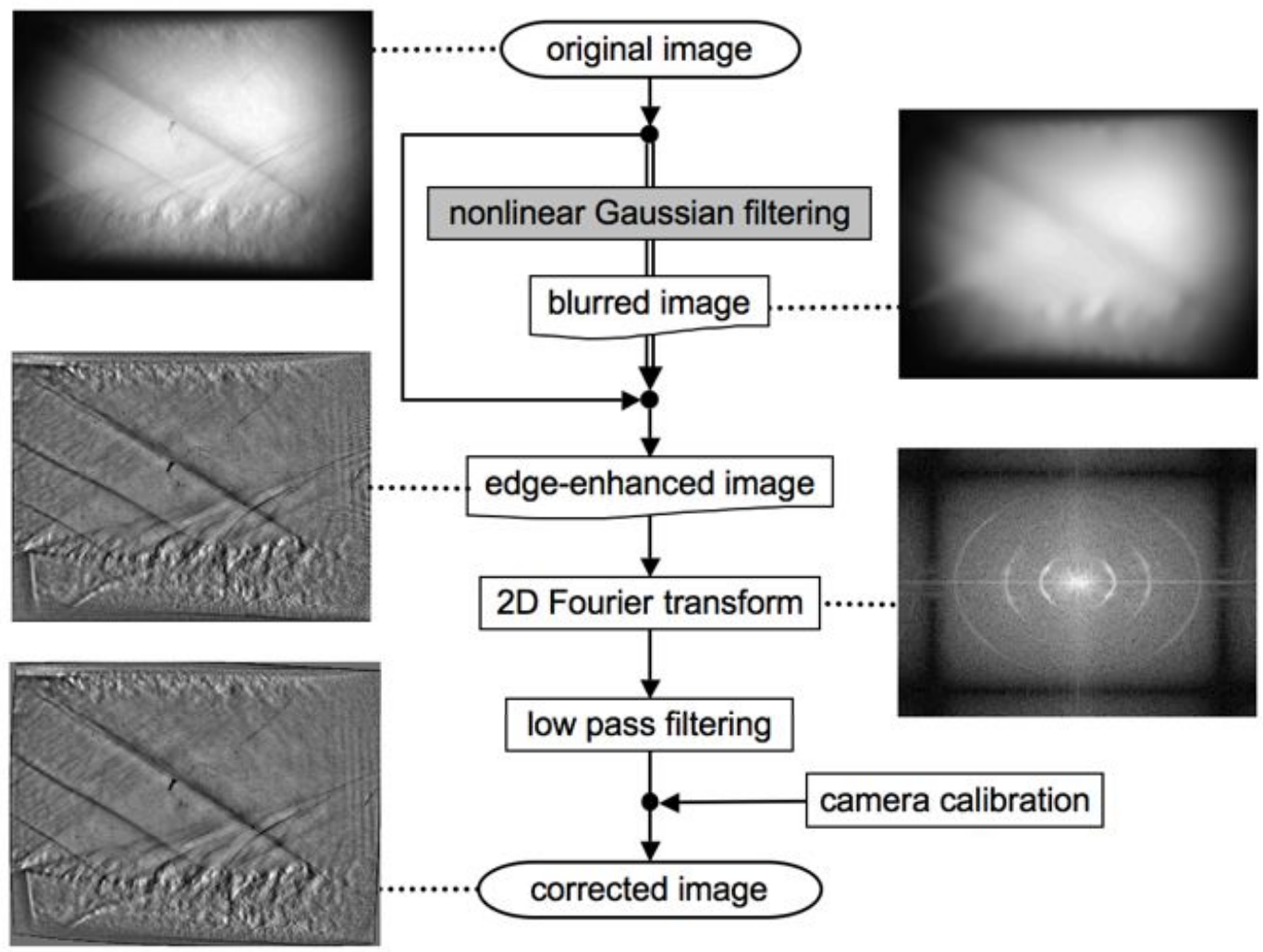

corrected image

Fig. 7 Flowchart of image processing.

\subsection{Uneven Illumination of Unprocessed Image}

Figure 8 shows typical unprocessed images in the cases of cold flow and combustion. In the case of cold flow, the center portion of the image well captures the flows features such as the shock 
waves and turbulent structures both in the wake behind the ramp and boundary layer on the upper wall. Appearance of the compression waves from the turbulent structures in the wake is the evidence that the present system had adequate sensitivity for the current application. Typical signal-to-noise ratio in the center portion of this image was about 7 . Note that the signal value was determined from the image intensities on the ramp shock and the noise was a spatial standard deviation of the image intensities taken for flow off.

In contrast with the center portion of the image, the image decreased in the brightness as ones goes to the edge of the field-of-view. This was corrected by "shade correction". Usually, blank (background) image is taken for flow off and it is subtracted or divided pixel by pixel from the flow image for shade correction. For the experiments of the under-expanded jet, we used this method to correct the background nonuniformity. This method, however, is unavailable for the images after the facility warm-up, because their illumination pattern was changed by the thermal distortion of the window glasses. Figure $8 \mathrm{~b}$ shows that the uneven illumination became noticeable even in the center portion of the image for the combustion case. A bright region appears on the upper right area in the image center, and the dark region appeared on the lower left area in the image center. The thermal distortion of the glass windows changed the shape of the source grid image on the cutoff grid plane. Mismatch of the grid line shapes between the source grid image and cutoff grid was the primary cause of the bright and dark regions in the image, as will be discussed in the Section 5.2. Similar bands appeared in the images of the cryogenic wind tunnel experiments (Gartenberg et al 1994). To correct this, we needed an alternative shade correction method.

a)

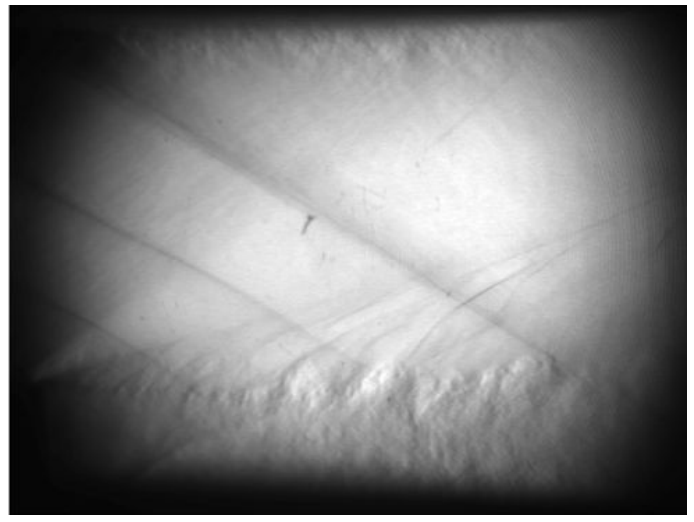

b)

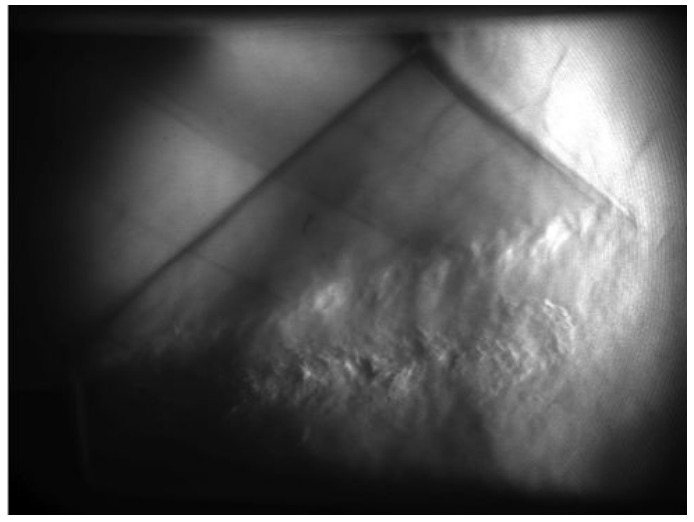

Fig. 8 Typical unprocessed images: a) cold flow case and b) combustion case. 


\subsection{Shade Correction}

The uneven illuminations on the images were removed without any additional acquisitions of the background image in this study. For the preliminary image processing, we used the ensembleaveraged image for the shade correction. The averaged image did not work well for the background illumination in the combustion case, because the bright and dark regions in the image, especially the jet region, temporally moved. Therefore, the background illumination was estimated from each flow image themselves. The wavelength of the background illumination pattern was smaller than those of the turbulent structures and shock waves. Therefore, a low pass filtering can extract the information on the background illumination from the flow image. The low pass filter removes detail in the image such as shock waves and turbulent structures and makes the image blurred. Dividing of the flow image by the blurred image corrects the background nonuniformity. This process also enhances the shock waves and turbulent structures. We applied a Gaussian low pass filter to estimate the background nonuniformity.

Figure 9 shows the effects of convolving Fig. 8a with the Gaussian filter increasing its standard deviation $\left(\sigma_{g}\right)$. The standard deviations are 31, 51, and 101 from left to right. Their kernel sizes were fixed to be $101 \times 101$ pixels. In Fig. 9, the shade corrected images by using these filtered images are also shown. The larger $\sigma_{g}$ smoothed the image to a greater extent. Therefore, the shade correction by using the larger $\sigma_{g}$ enhanced the larger flow structures as shown in the series of images in Fig. 9. The scales of enhanced structures could be selected by changing in $\sigma_{g}$. The series of images in Fig. 9 shows that relatively large $\sigma_{g}(\geq 51)$ is required to capture the moderate size flow structures. However, overexposed and underexposed regions appeared near the wall for large $\sigma_{g}$, because the image intensity suddenly changed across the boundary of the walls. These overexposed and underexposed regions obstructed the details of the flow structures near the wall.

We solved this problem by locally changing $\sigma_{g}$ in the image. The local values of $\sigma_{g}$ were determined from the static measure of the image intensity in the interrogation area where the Gaussian kernel was convoluted. If the walls are sited in the interrogation area, the spatial averaged intensity $(\hat{I})$ in this region will be lower and its spatial standard deviation $(\hat{o})$ will be higher than that in the flow region, because the image intensity suddenly changed across the 
boundary of the walls. Therefore, we determined $\sigma_{g}$ as following equation so that $\sigma_{g}$ was decreased near the wall.

$$
\begin{gathered}
\sigma_{g}=\alpha \cdot \frac{\hat{I}}{\hat{\sigma}} \\
\hat{I}=\frac{1}{p k^{2}} \sum_{j=1}^{p k} \sum_{i=1}^{p k} I_{i j} \\
\hat{\sigma}=\sqrt{\frac{1}{p k^{2}} \sum_{j=1}^{p k} \sum_{i=1}^{p k}\left[I_{i j}-\hat{I}\right]^{2}}
\end{gathered}
$$

where $I_{i j}$ is the image intensity of $i j$ pixels where the filter is convoluted, $p k$ is the kernel size of the filter, and $\alpha$ is control parameter which determined the scales of the recognized structures. The optimal $\alpha$ is dependent on the scale in the image. We found that $\alpha$ of 3 was adequate by trial and error. This value yielded $\sigma_{g} \sim 80$ in the shear layer behind the ramp and $\sigma_{g} \sim 20$ near the wall. The value of $\alpha$ was fixed in all cases in present study.

The values $\hat{I}$ and $\hat{o}$ depended on the kernel size of $p k$. The value of $p k$ was determined by recursive approach using $\sigma_{g}$. The initial $p k$ was set to be 51 for the present study. This yielded initial $\hat{I}, \hat{O}$ and $\sigma_{g}$. Once $\sigma_{g}$ was calculated, the kernel size was changed as the following equation.

$$
p k_{\text {new }}=\frac{1}{2}\left(p k_{\text {old }}+\left(2 \sigma_{g}+1\right)\right)
$$

The new $\hat{I}, \hat{o}$ and $\sigma_{g}$ were recursively calculated with inputting new $p k$. We found that four times iteration sufficiently converged the values of $p k$ and $\sigma_{g}$.

\subsection{Noise Reduction}

The shade correction enhanced not only the flow structures but also the concentric circle fringes on the Fresnel screen as shown in Fig. 9. A frequency filter was applied to eliminate this and to increase the signal-to-noise ratio of the images. Two-dimensional Fourier transform converted the image into wave number spectrum. Amplitude spectrum of the shade corrected image is shown in Fig. 7 with logarithmic scale. Three concentric circles appeared in this image. These originated from the Fresnel patterns in the background of the enhanced image. The diameter of the minimum circle was 0.1 pixel $^{-1}$ in Fourier domain. This means that the Fresnel patterns appeared at each 10 pixel in the image. This figure also tells us that almost all image information 
was concentrated within low wave number region smaller than the diameter of the minimum circle.

Therefore, we applied the low pass filter to pass only this region.
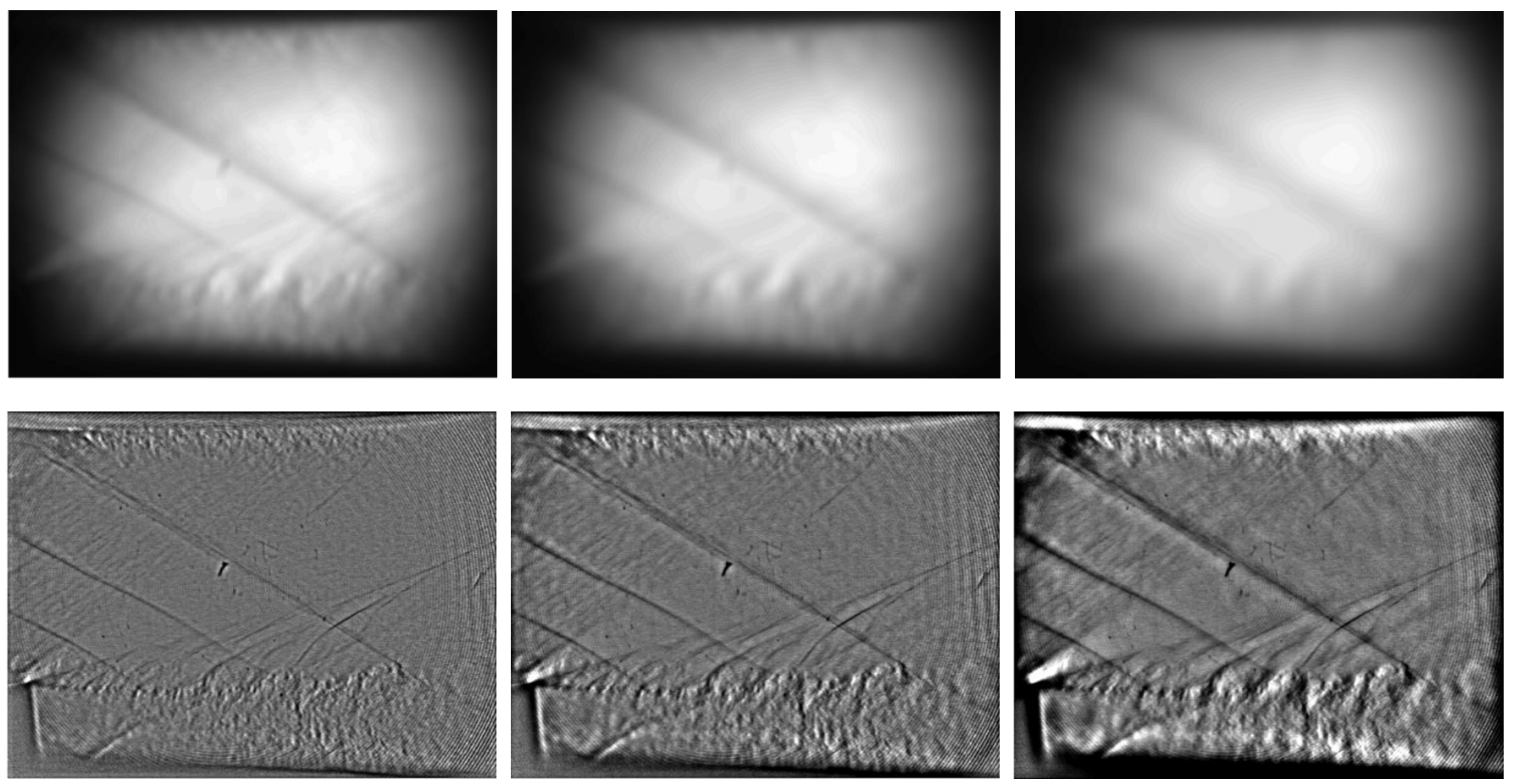

Fig. 9 Demonstration of convoluting Fig. 8a with the Gaussian filters having three different standard deviations (upper part) and the shade corrected image divided by them (lower part).

\subsection{Image Deformation}

The present imaging system used the Fresnel lens for the analyzer assembly. A small missalignment of the Fresnel lens could distort the image. This was corrected by the image deformation process. The calibration image was acquired before the test run and was used to dewarp the flow images. The calibration target composed of a matrix of $2.5 \mathrm{~mm}$ equally spaced dots with $1 \mathrm{~mm}$ diameter. This image deformation, however, is ineffective in the window distortion originating from the heating of the window glass, because the calibration image cannot be captured after facility warm-up. As will be mentioned in the Section 5.2, the facility warm-up induced the mechanical thermal expansion of the facility and changed the position of the injector ramp. The angles of both the diverging wall and ramp, however, were unchanged in the deformed image even after the facility warm-up. Therefore, we believe that the thermal distortion of the glass less

\section{affected the image deformation.}

The final processed image is shown in Fig. 7. This figure shows that the current postprocessing obtained a much more uniform background and high signal-to-noise ratio and made the 
flow structures clear. For all the results presented below, only the post-processed schlieren images are included.

\section{Flow Visualization Results}

Once the performance of the focusing schlieren system was understood using the underexpanded jet, the salient dual-mode scramjet flow features were identified at a cold flow case with $T_{0}=300 \mathrm{~K}$. Attention was then turned to the process of warming up the facility before the final test condition with $T_{0}=1200 \mathrm{~K}$ was considered in the cases of ignition and steady combustion.

\subsection{Cold Flow Case}

Figure 10 shows typical images in the case before the facility warm-up. Figure 10a is the image in the case of fuel off and Fig. 10b is that of fuel-air mixing. For reference, the downstream end of the ramp is in the lower left corner of the image. The combustor walls including the injector ramp are masked out in this figure. The mainstream flows from left to right in these pictures. In both cases, the schlieren images clearly captured shock waves and turbulent structures. In addition, the images well captured the pressure waves due to the turbulent eddy convection in the shear layer.

In the case of fuel off, very large turbulent eddies were generated at $x / H>3$ in the wake developed downstream the ramp base. Compression waves were emitted from these structures. Many of these waves merged with each other and formed a recompression shock wave with an angle of $12^{\circ}$ to the wall on the constant-area section. The shape of the compression waves at $x / H \sim$ 4 was like a bow shock wave. This means that the convection velocity of the turbulent eddies was slower than that of mainstream at this point. The turbulent structures also can be seen near the diverging wall at $x / H>2$ in the wake. The size of the structures near the wall was smaller than that of the outer edge of the wake. The shock wave from the leading edge of the ramp reflected on the upper wall at $x / H \sim 0$ with an angle of $-32^{\circ}$. This shock wave impinged into the wake at $x / H \sim 5$. This agrees with the trend of $P_{W}$ in Fig. 2. Mach number was estimated to decrease from 2 to 1.6 behind the reflected shock wave.

In the case of fuel on, two shock waves appeared in the mainstream due to the fuel injection. One was anchored at the trailing edge of the ramp and the other was anchored at the Mach disk position of the first shock cell in the jet. Only a single under-expanded shock cell structure is visible in the fuel jet. These shock waves were generated by changes in the flow angle and were 
associated with the shock cell structure. The angle of the former shock wave was $21^{\circ}$ and that of the latter shock wave was $23^{\circ}$ to the wall on the constant-area section. The latter shock wave seems to be stronger than the former one judging from their image intensity. The turbulent eddies in the jet appear to develop downstream of the barrel shock. The size of the turbulent eddies in the jet were smaller than those in the wake in the case of fuel off. It can be seen that many compression pressure waves were associated with the fuel injection. The shape of these compression waves appears like spherical waves that are conveyed downstream. The shape of these waves is different from that in the case of fuel off. In the case of fuel on, the turbulent eddies in the jet could move faster than the mainstream, because the sonic speed of hydrogen fuel is much faster than the mainstream. Note that the image in the center part of the fuel jet was slightly blurred. This is due to the focusing effect of the system. The present system had quite small $D O F$ less than the ramp width. The turbulent structures are mainly developed in the shear layer between the jet and mainstream. If the jet expands over the ramp width, the structures in the spanwise direction will be out of focus. Therefore, this blurred region is the evidence that the jet expanded over the ramp width here.
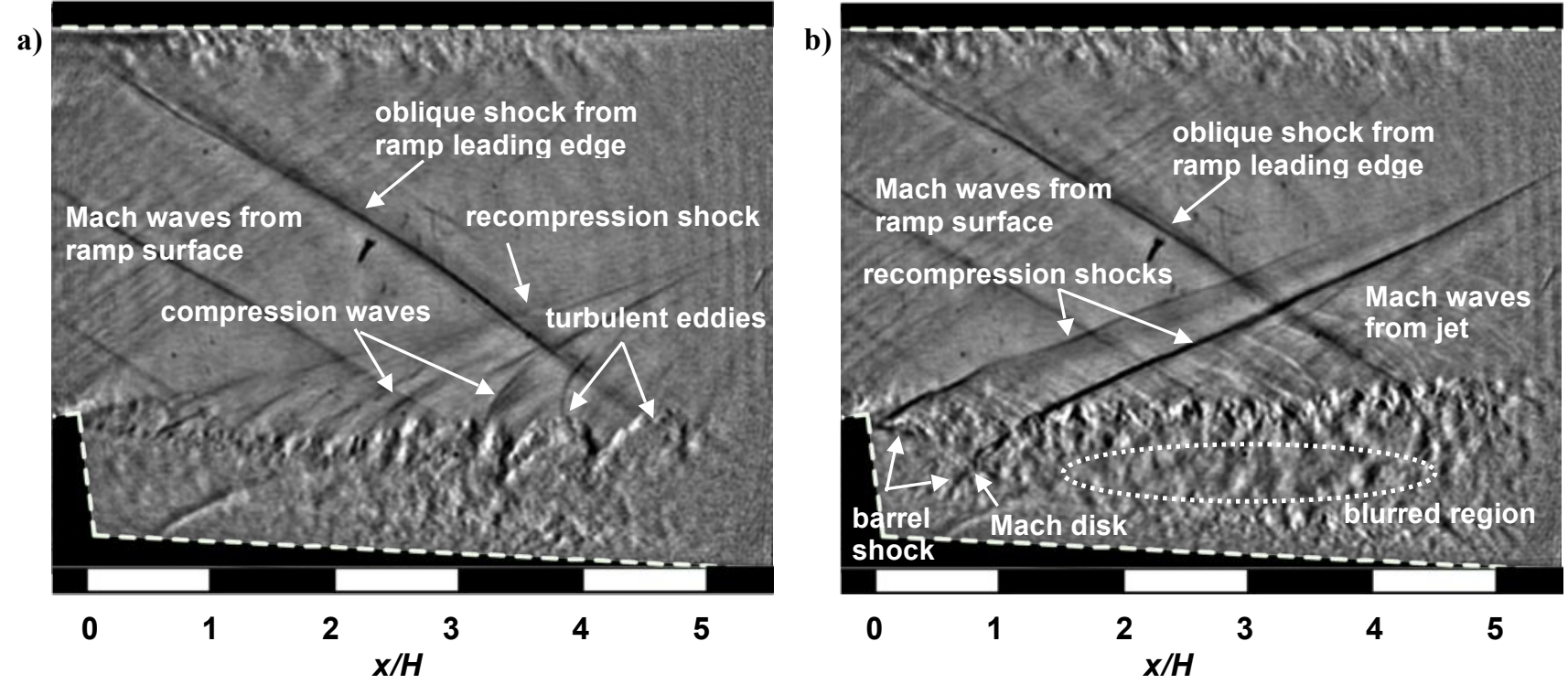

Fig. 10 Typical focusing-schlieren images under cold flow condition: a) fuel off and b) fuel on.

\subsection{Facility Warm-Up Case}

Figure 11 shows the images in the case of fuel off during the facility warm-up process. The images were taken at $T_{0}=600 \mathrm{~K}$ (Fig. 11a), $900 \mathrm{~K}$ (Fig. 11b), and $1200 \mathrm{~K}$ (Fig. 11c). For these cases, the cutoff grid position was held constant. The cutoff grid position was slightly realigned 
after taking Fig. 11c and before taking Fig. 11d. Figure 11d was taken at the same $T_{0}$ condition of Fig. 11c. For reference, the dashed lines in these images depict the wall and ramp positions before the facility warm-up.

The series of images in Fig. 11 shows that the ramp position changed as $T_{0}$ was increased. Change of the wall position from before to after the facility warm-up was due to the mechanical thermal expansion of the facility. The shape of the ramp and the angle of the diverging wall, however, were not changed even after the facility warm-up. This series of images in Fig. 11 are the evidence that the image distortion due to the facility warm-up was relatively small. This is a validation of the experimental approach adopted for the present study.

Figure 11 also indicates that the image sensitivity decreased with increasing $T_{0}$. The sensitivity, however, recovered with the realignment of the grid position even with the highest $T_{0}$. The stagnation temperature up to $T_{0}=600 \mathrm{~K}$ had minimal effects on the sensitivity. Figure $11 \mathrm{a}$ was essentially similar to Fig. 10a. When the stagnation temperature reached $900 \mathrm{~K}$, the image sensitivity slightly declined. For example, the turbulent structures in the boundary layer on the upper wall and the pressure waves from the ramp surface almost disappeared. However, the sensitivity was still high enough to capture the turbulent structures in the wake behind the ramp and the pressure waves from these structures.

When the stagnation temperature reached $1200 \mathrm{~K}$, the image was drastically altered as shown in Fig. 11c. Figure 11c shows that all the flow structures disappeared. For this case, the image intensity of the unprocessed image greatly increased compared with those of Fig. 11b. These drastic changes were mainly attributed to the translation of the source grid images in its focal plane due to the thermal distortion of the window glass. Heating of the window glasses changed their refractive index. This can vertically and laterally translate the grid images in its focal plane. In addition, this can deform the line shapes of the grid images. The translation and deformation of the grid images would decrease the degree of cutoff by the cutoff grid. This is the most probable reason why all the flow structures disappeared in Fig. 11c.

Based on these findings, the cutoff grid position was translated perpendicular to the direction of its gridlines to improve the sensitivity, so that the degree of cutoff was increased. Only this realignment recovered the image sensitivity even at $T_{0}=1200 \mathrm{~K}$ as shown in Fig. $11 \mathrm{~d}$. This image captured not only the shock waves but also turbulent structures in the wake. The image also captured the relatively strong pressure waves due to the turbulent eddy motion. The sensitivity in 
Fig. 11d was similar in level to that in Fig. 11b. Thus, the present system still had enough sensitivity to investigate the flow features in the dual-mode scramjet at the test point of $T_{0}=1200$ $\mathrm{K}$ that was required for combustion.

If the imaging system had much wider $D O F$, additional realignment of the cutoff grid position would be required to recover the sensitivity. For the preliminary experiment, we used the focusing schlieren system having unsharp DOF of $\pm 20 \mathrm{~mm}$. For this system, we needed to move the cutoff grid position to the direction of the optical axis as well as those in the focal plane, to increase the system sensitivity after the facility warm-up. For the system having much wider $D O F$, the thermal distortion of the glasses remarkably moved the focal point of the grid image to the direction of the optical axis. Even with these realignments of the cutoff grid, the preliminary system was not able to capture the detail flow structures in the combustor after the facility warmup.

As a final note on cutoff grid realignment, comparison of Fig. 10a and Fig. 11d implied that the realignment of the cutoff grid was not perfect. This is attributed to the deformation of the gridline shapes due to the thermal distortion of the glasses. The high sensitivity of the focusing schlieren system requires not only the proper alignment of the cutoff grid position but also the proper line shapes of the cutoff grid aligned with the source grid images. Therefore, the cutoff grid was photographically made as mentioned previously. Before the facility warm-up, the photographically made cutoff grid accurately coincided with the source grid image, resulting in the high sensitivity shown in Fig. 10. The source grid image, however, was deformed by the facility warm-up and its lines shape slightly differed from those of the cutoff grid which was made before the facility warm-up. This mismatch is attributed to the uneven illumination across the field of view in the unprocessed image at the test point of $T_{0}=1200 \mathrm{~K}$. 
a)

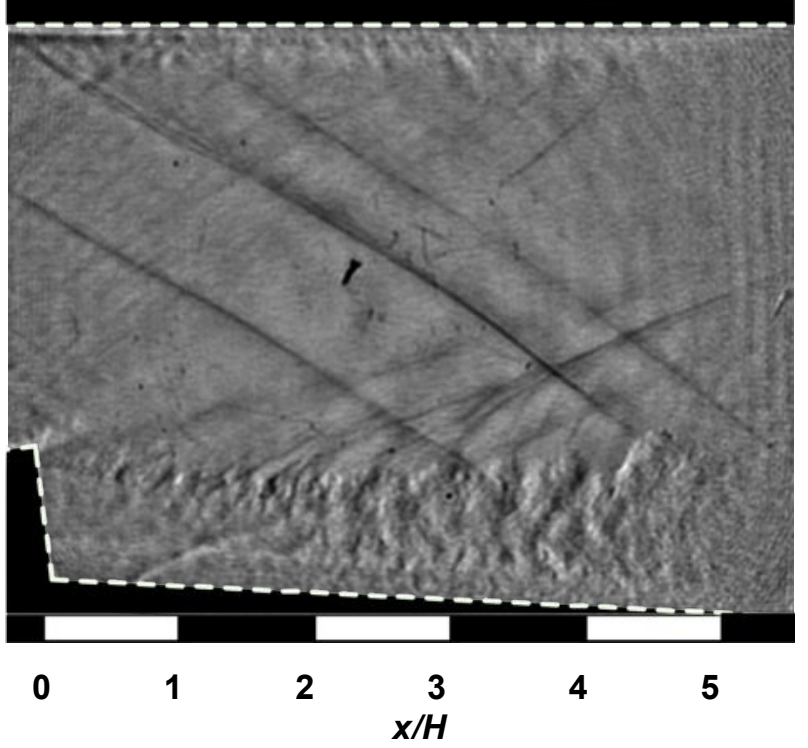

c)

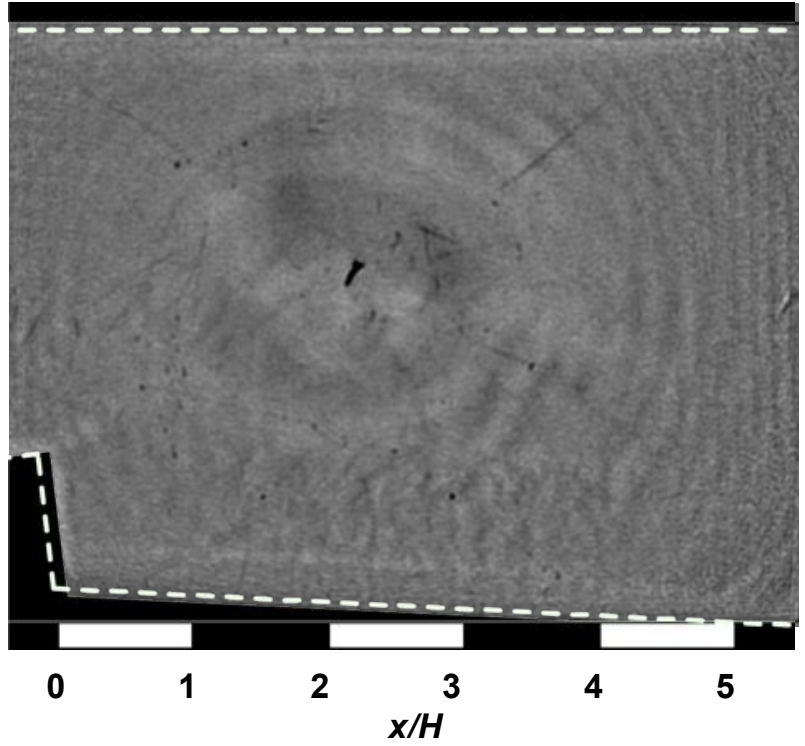

b)

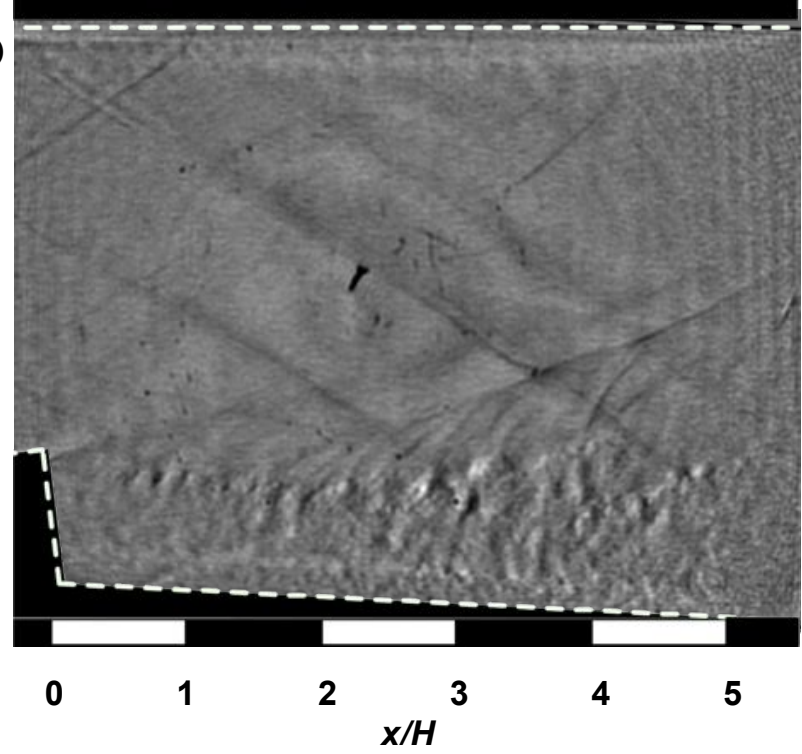

d)

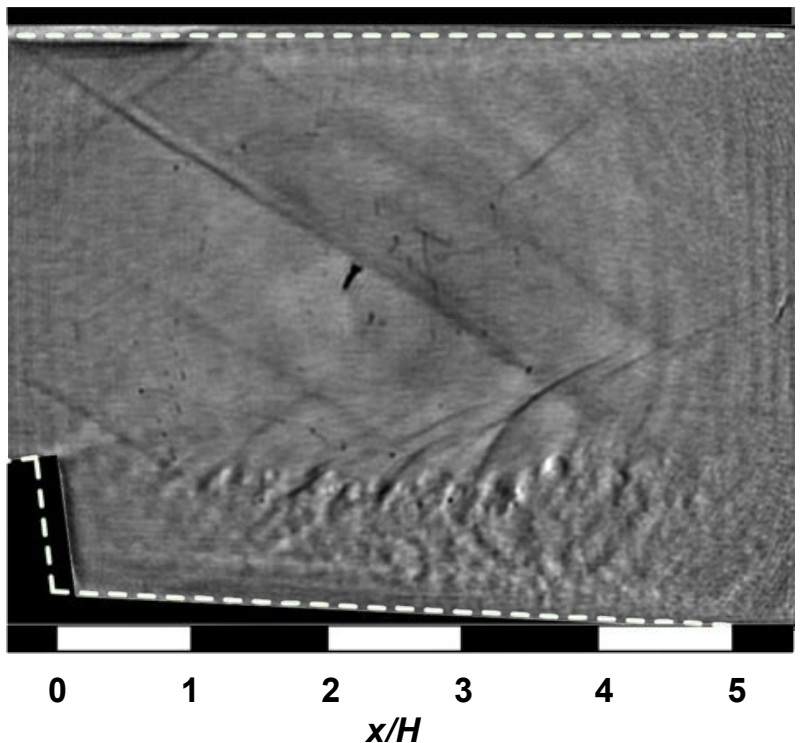

Fig. 11 Effect of facility warm-up on sensitivity of focusing-schlieren: a) $\left.T_{0}=600 \mathrm{~K}, \mathrm{~b}\right) 900 \mathrm{~K}$, c) $1200 \mathrm{~K}$, and d) $1200 \mathrm{~K}$ with cut-off grid adjustment.

\subsection{Ignition Case}

Figure 12 shows typical images that were acquired during the fuel-air ignition process. This process was undertaken once the facility was at the $T_{0}=1200 \mathrm{~K}$ condition. The igniter consisted of a hydrogen-oxygen detonation tube that expelled hot combustion products into the scramjet flowpath via a flush-wall, 90-degree, sonic injection port. This port was located approximately four ramp heights downstream of the ramp base. Figure $12 \mathrm{a}$ is an image captured just prior to ignition (with fuel flow established) and Fig. 12b is that after ignition. The time interval between

Figs. $12 \mathrm{a}$ and $12 \mathrm{~b}$ was $0.1 \mathrm{~s}$. In both cases, the overall fuel equivalence ratio was 0.2 and the 
igniter was operating. Before ignition, the mainstream remained at supersonic speed. The flow structure is similar to that in the cold flow case (Fig. 10b) except for the flowfield around the igniter. The presence and effect of the igniter jet are clearly seen at $x / H \sim 4$. The igniter jet penetrated into the outer edge of the fuel jet, though the fuel jet did deflect it downstream.

After ignition, the flow structure drastically changed. Combustion changed the shape and angle of the mixing layer between the fuel jet and mainstream air. The volumetric expansion due to combustion inclined the mixing layer with an angle of $18^{\circ}$ to the wall on the constant-area section. This angle was steeper than the ramp angle. As a result, the strong shock wave was generated at the trailing edge of the ramp. Impingement of the combustion-generated shock wave on the upper wall induced the boundary layer separation and formed a bifurcated $x$-shaped shock wave indicated in Fig. 12b. The angle of this bifurcated shock wave was about $42^{\circ}$ to the upper wall. From image to image, this shock wave highly oscillated and changed its position.

The shape of the igniter jet also changed due to the pressure rise. The igniter jet more deeply penetrated across the fuel jet and generated the bow shock wave in the mainstream behind the $x$ shaped shock wave.

a)

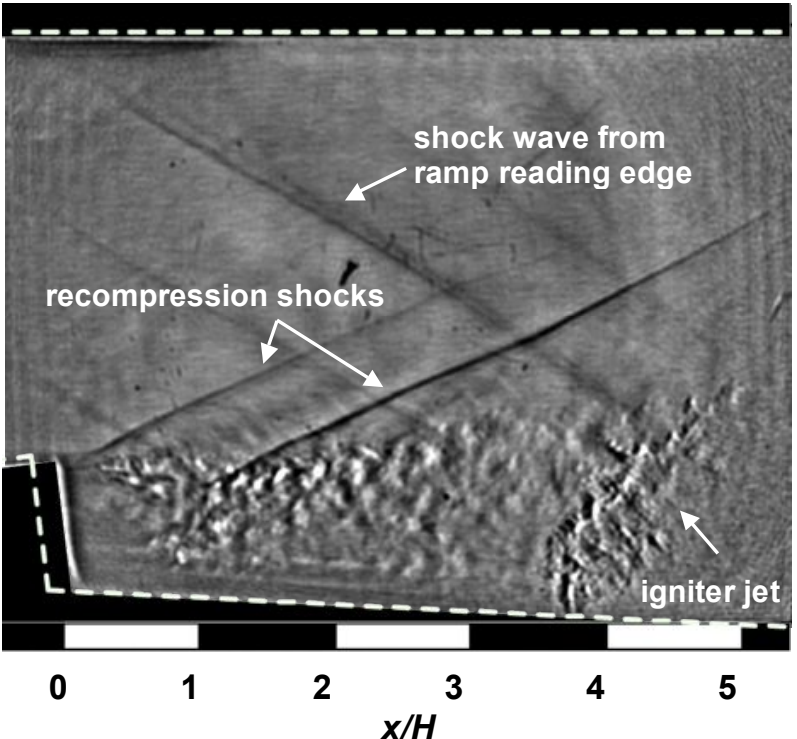

b)

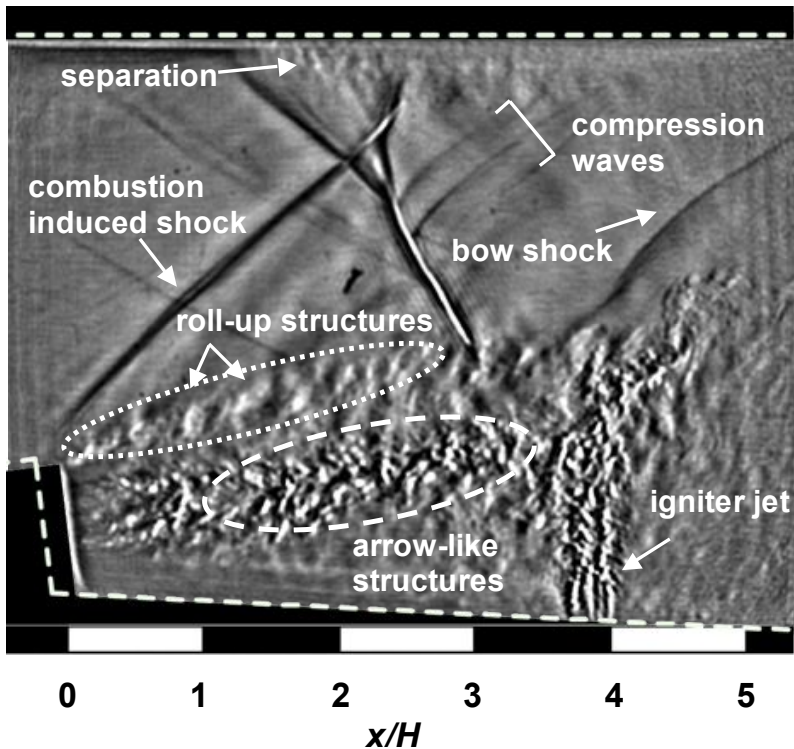

Fig. 12 Typical focusing-schlieren images in the case with igniter on: a) before flowpath ignition and b) after flowpath ignition.

\subsection{Combustion case}

Figure 13 shows a typical image in the case of fuel-air reacting at $\Phi=0.17$. Here the igniter system was off, but combustion was steady and self-sustaining. The flow features in this case are 
similar to those in Fig. 12b that was immediately taken after ignition. The major difference between Figs. 13 and $12 \mathrm{~b}$ is both the position and angle of the combustion-generated shock wave. This shock wave is positioned downstream, compared with Fig. 12b, due to a lower $\Phi$. The shock angle in this case was about $38^{\circ}$ to the wall on the constant-area section. In sequences of images, the shock wave was seen to be highly oscillatory. The image shows the oblique shock wave as similar to Fig. 8 b, but other images showed the $x$-shaped shock wave as similar to Fig. 12b. This combustion-induced shock wave was observed to be stronger than the shock wave from the leading edge of the ramp, because the signal level on this shock wave was higher than that on the ramp shock.

The fuel jet axis was parallel to the ramp face in Fig. 13. A mixing layer developed between the mainstream and the fuel plume. The roll-up structures appeared in this layer. These structures are more visible in Fig. 12b. These structures indicate that the Kelvin-Helmholtz instability occurred in this layer. This implies that the convective Mach number in the mixing layer was low. Figure 13 also shows that the flow structures near the diverging wall and those between the mixing layer and the core of the fuel plume were blurred. On the other hand, they were clearly visible in the core of the fuel plume. Their shapes look like arrows (jet like). This indicates that the flow speed in the core of the fuel plume was higher than the surrounding one. The first shock cell in the jet is barely visible, but the other shock cell in the jet is invisible. The turbulent structures masked the shock cells at the downstream location. Averaging of 300 instantaneous images revealed that four shock cells existed in the jet.

Based on these measurements, it can be concluded that the focusing schlieren system successfully visualized the flowfield in the dual-mode scramjet combustor. The application of the focusing-schlieren system to direct-connect scramjet testing has enabled the acquisition of the first-of-their-kind measurements. The images have enabled a better understanding of the fuel-air mixing, ignition and combustion behavior. The present measurements, and the broader database, not only help understand the flow but also serve as a valuable resource for numerical model validation. 


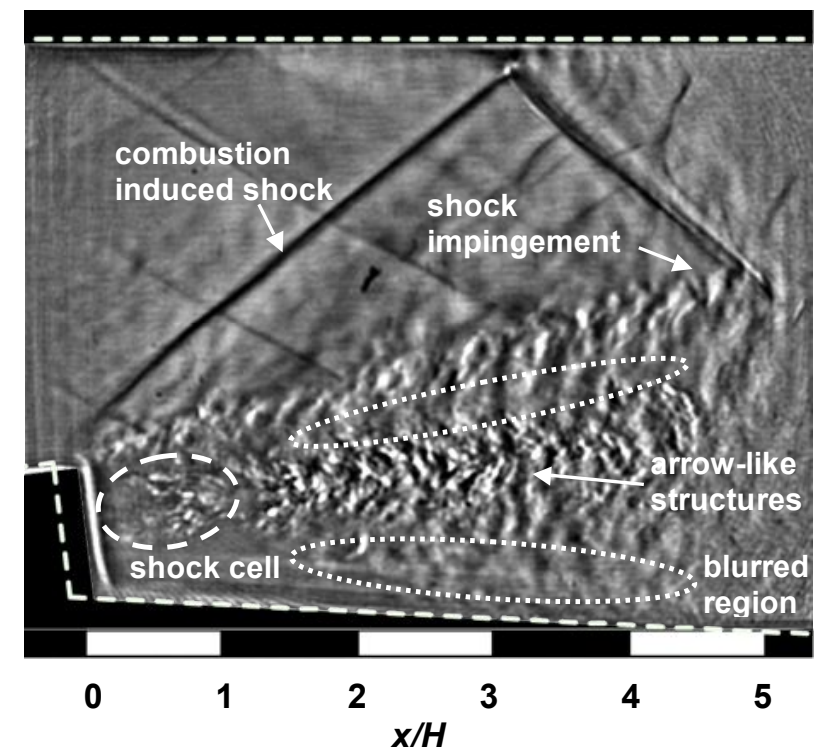

Fig. 13 Typical focusing-schlieren image in the case of fuel-air reacting.

\section{Conclusion}

A focusing-schlieren system, with a $\pm 5 \mathrm{~mm}$ depth of focus, was developed to visualize the flowfield in a direct-connect dual-mode scramjet. This system was applied to an electrically heated, continuous flow wind tunnel simulating a Mach 5 flight condition with a stagnation temperature of $1200 \mathrm{~K}$.

The system was used to successfully visualize the flowfield, though the image sensitivity did decline somewhat with the facility warm-up. For the cold flow experiments, the system captured not only shock and expansion waves but also turbulent structures in the flowfield. With increasing flow stagnation temperature, the image sensitivity declined due to the thermal distortion of the windows. All flow structures captured in the cold flow experiment disappeared when the stagnation temperature reached $1200 \mathrm{~K}$, if the cutoff grid position was not realigned. Slight realignment of the grid position recovered the sensitivity enough to capture the turbulent structures in the fuel jet.

The imaging captured the change of the flowfield due to fuel-air ignition and combustion process. Combustion drastically changed the flowfield due to volumetric expansion of the fuel jet. Combustion-generated pressure rise pushed up a bifurcated shock wave up to the trailing edge of the ramp fuel injector. This shock wave highly oscillated, and its shape temporally changed. The turbulent structures in the fuel jet were arrows in the shape. The convection speed of these structures appeared to be higher than that of the undisturbed Mach 2 mainstream. Roll-up 
structures appeared in the mixing layer between the fuel jet and mainstream. This was evidence of a Kelvin-Helmholtz instability affecting the fuel-air mixing process in the dual-mode scramjet.

Acknowledgments The authors appreciate the assistant of Dr. Roland Krauss and Mr. Roger Reynolds in operating the supersonic combustion facility. This work was supported by Young Researcher Oversea Study Program of Japan Society for the Promotion of Science. This research was also supported by the National Center for Hypersonic Combined Cycle Propulsion grant FA 9550-09-1-0611. The technical monitors on the grant were Chiping Li (AFOSR) and Aaron Auslender and Rick Gaffney (NASA).

\section{References}

Babinsky H, Harvey JK (2011) Shock Wave-Boundary Layer Interactions. Cambridge University press, New York 55-56.

Ben-Yakar A, Hanson RK (2002) Ultra-Fast-Framing Schlieren System for Studies of the Time Evolution of Jets in Supersonic Crossflows. Exp Fluids 32:652-666. doi: 10.1007/s00348002-0405-z

Ben-Yakar A, Mungal MG, Hanson RK (2006) Time evolution and Mixing Characteristics of Hydrogen and Ethylene Transverse Jets in Supersonic Crossflow. Phys Fluids 18:026101-1026101-16. doi: 10.1063/1.2139684

Brown GL, Roshko A (1974) On Density Effects and Large Structure in Turbulent Mixing Layers. J Fluids Mech 64(4):775-816. doi: 10.1017/S002211207400190X

Fotia ML, Driscoll JF (2012) Isolator-Combustor Interactions in a Direct-Connect RamjetScramjet Experiment. J Prop Power 28(1): 83-95. doi: 10.2514/1.B34367

Gartenberg E, Weinstein LM, Lee Jr EE (1994) Aerodynamic Investigation with Focusing Schlieren in a Cryogenic Wind Tunnel. AIAA J 32(6): 1242-1249. doi: 10.2514/3.12126

Hargather M, Lawson M, Settles GS, Weinstein LM (2011) Seedless Velocimetry Measurements by Schlieren Image Velocimetry. AIAA J 49(3): 611-620. doi: 10.2514/1.53170

Johansen CT, McRae CD, Danehy P, Gallo E, Cantu L, Magnotti G, Cutler A, Rockwell RD, Goyne CP, McDaniel JC (2012) OH PLIF Visualization of the UVa Supersonic Combustion Experiment: Configuration A. AIAA-2012-2887. doi: 10.2514/6.2012-2887

Kouchi T, Hoshino T, Sasaya S, Masuya G (2009) Time-Space Trajectory of Unsteady Jet into Supersonic Crossflow Using High-Speed Framing Schlieren Images. AIAA-2009-7316. doi: $10.2514 / 6.2009-7316$

Kouchi T, Masuya G (2012), Quantification of Convection Velocity and Dominant Scale of Large-Scale Structures by High-Speed Schlieren Imaging. AIAA-2012-4148. doi: $10.2514 / 6.2012-4148$ 
Krauss RH, McDaniel JC (1992) A Clean Air Continuous Flow Propulsion Facility. AIAA92-3912. doi: 10.2514/6.1992-3912

Laurence SJ, Schramm JM, Karl S, Hannemann K (2011) An Experimental Investigation of Steady and Unsteady Combustion Phenomena in the HyShot II Combustor. AIAA-2011-2310. doi: $10.2514 / 6.2011-2310$

Matsuo K, Miyazato Y, Kim HD (1999) Shock train and pseudo-shock phenomena in internal gas flows. Progress in Aerospace Sciences 35(1):33-100. doi:10.1016/S03760421(98)00011-6

Rockwell RD, Goyne CP, Rice BE, Tatman BJ, Smith C, Kouchi T, McDaniel JC, Fulton JA, Edwards JR (2012) Close-collaborative Experimental and Computational Study of a Dual-mode Scramjet Combustor. AIAA-2012-0113. doi: 10.2514/6.2012-113

Settles GS (2001) Schlieren and Shadowgraph Techniques. Springer-Verlag, New York, pp 88-103.

Weinstein LM (2010) Review and Update of Lens and Grid Schlieren and Motion Camera Schlieren. Euro Phys J 182:65-95. doi: 10.1140/epjst/e2010-01226-y

Weiss A, Grzona A, Olivier H (2010) Behavior of shock trains in a diverging duct. Exp Fluids 49:355-365. doi: 10.1007/s00348-009-0764-9 